\author{
Pre-and post-partum nutritional management to optimise fertility in high-yielding dairy cows in \\ confined TMR systems \\ J K Drackley and F C Cardoso \\ Department of Animal Sciences, University of Illinois, 1207 West Gregory Drive, Urbana, Illinois, USA \\ Email: drackley@illinois.edu
}

Implications Nutritional strategies and feeding management during pre-calving and post-calving periods impact health, productivity and fertility of high producing dairy cows. Formulating diets to meet requirements of the cows but avoid overconsumption of energy may improve outcomes of the transition period and lead to improved fertility. Management to improve cow comfort and ensure good intake of the ration is pivotal for success. Impacts of the transition programme should be evaluated in a holistic way that considers disease occurrence, productivity and fertility.

\begin{abstract}
The 6-8 week period centered on parturition, known as the transition or periparturient period, is critical to welfare and profitability of individual cows. Fertility of high producing cows is compromised by difficult transitions. Deficiencies in either nutritional or non-nutritional management increase risk for periparturient metabolic disorders and infectious diseases, which decrease subsequent fertility. A primary factor impeding fertility is the extent of negative energy balance (NEB) early postpartum, which may inhibit timing of first ovulation, return to cyclicity, and oocyte quality. In particular, pronounced NEB during the first 10 days to 2 weeks (the time of greatest occurrence of health problems) is critical for later reproductive efficiency. Avoiding over-conditioning and preventing cows from over-consuming energy relative to their requirements in late gestation result in higher dry matter intake (DMI) and less NEB after calving. A pooled statistical analysis of previous studies in our group showed that days to pregnancy are decreased (by 10 days) by controlling energy intake to near requirements of cows before calving compared with allowing cows to over-consume energy. To control energy intake, total mixed rations (TMR) must be well balanced for metabolisable protein, minerals and vitamins yet limit total DM consumed, and cows must uniformly consume the TMR without sorting. Dietary management to maintain blood calcium and rumen health around and after calving also are important. Opportunities may exist to further improve energy status in fresh cows. Recent research to manipulate the glucogenic to lipogenic balance and the essential fatty acid content of tissues are intriguing. High-producing cows that adapt successfully to lactation can have high reproductive efficiency, and nutritional management of the transition period both pre- and post-calving must facilitate that adaptation.
\end{abstract}

Conclusion In TMR systems, formulation and delivery of appropriate diets that limit total energy intake to requirements but also provide proper intakes of all other nutrients before calving can help lessen the extent of NEB after calving. Effects of such diets on indicators of metabolic health are generally positive, suggesting the potential to lessen effects of periparturient disease on fertility. Strategies for diet formulation to improve DMI and lessen NEB of fresh cows are less well researched, but the balance between adequate physically effective fiber and starch fermentability is critical. Continued development of innovative strategies such as manipulation of glucogenic to lipogenic nutrients and essential fatty acid supply should pay dividends to fertility. 


\section{Pre-and post-partum nutritional management to optimise fertility of dairy cows in pasture-based systems \\ S T Butler \\ Teagasc, Animal and Grassland Research and Innovation Centre, Moorepark, Fermoy, Co. Cork, Ireland \\ Email:stephen.butler@teagasc.ie}

Implications Achieving the appropriate BCS at parturition and minimizing BCS loss are the overarching goals of nutritional management in pasture-based system. A large number of systems studies have demonstrated that simply adding more concentrate will not result in improved fertility. Future research needs to identify specific micro- and macro-nutrients that can stimulate components of the reproductive axis.

Abstract The efficiency of milk production in pasture-based systems is heavily influenced by calving pattern, necessitating excellent reproductive performance in a short breeding season. Where grazed pasture is the major component of the diet, cows are underfed relative to their intake potential. The cow responds by reducing milk output, but fertility is generally better than high intake confinement systems that achieve greater milk production per cow. A number of studies have identified body condition score (BCS) measurements that are related to likelihood of both submission and conception. Blood metabolites and metabolic hormones linked to fertility outcomes are now well characterized. In general, fertility variables have favourable associations with circulating concentrations of glucose, insulin and insulin-like growth factor-1 and unfavourable associations with non-esterified fatty acids, beta-hydroxybutyrate and endogenous growth hormone. Nutritional strategies to impact these metabolic indicators have been utilised, but effects on herd fertility are inconsistent. Simply supplementing cows with additional energy in the form of standard concentrates does not appear to have a pronounced effect on fertility. Energy from additional concentrates fed during lactation is preferentially partitioned towards extra milk production rather than BCS repletion. The higher the genetic merit for milk production, the greater the partitioning of additional nutrients to the mammary gland. This review outlines the unique nutritional challenges of pasturebased systems, the role of specific metabolic hormones and metabolites in regulating reproductive function, and nutritional strategies to improve herd fertility.

Conclusions Successful pasture-based systems of milk production are reliant on achieving excellent fertility performance, thereby allowing a compact calving period that coincides with the onset of spring pasture growth. Nutritional management has a key role to play in achieving fertility targets. It is now firmly established that achieving the correct BCS at calving (3.00 to 3.25 ) and minimizing postpartum BCS loss ( $\leq 0.5$ BCS units) are key drivers of herd fertility performance, and nutritional management must revolve around achieving these goals. Development of nutritional strategies to favourably impact metabolic indicators that regulate the reproductive axis should be prioritised. 


\title{
Optimising reproductive performance of beef cows and replacement heifers
}

M G Diskin $^{1}$ and D A Kenny ${ }^{2}$

Animal and Bioscience Research Department,

${ }^{1}$ Teagasc, Animal \& Grassland Research and Innovation Centre, Athenry, Co. Galway. Ireland.

${ }^{2}$ Teagasc, Animal \& Grassland Research and Innovation Centre, Grange, Dunsany, Co. Meath. Ireland.

Email:michael.diskin@teagasc.ie

Implications Reproductive efficiency is key to the biological and economic sustainability of suckled calf enterprises. A thorough understanding of the many interacting factors, including genetics, nutrition, body energy reserves, health and semen/bull fertility is required to optimise overall cow productivity. The lifetime productivity of the beef bred female will commence from the onset of puberty and be dictated by subsequent critical events including age at first calving, duration of the postpartum interval for each successive calving and ultimately length of intercalving intervals. Optimum nutritional management is required at all stages of the production cycle to ensure the timeliness of these events together with vigilance for adequate male fertility and, or appropriate heat detection and insemination technique.

\begin{abstract}
A reproductively efficient beef cow herd will be fundamental to meeting the protein and specifically, red meat demand of an ever increasing global population. However, attaining a high level of reproductive efficiency is underpinned by producers being cognisant of and achieving many key targets throughout the production cycle and requires significant technical competency. The lifetime productivity of the beef bred female commences from the onset of puberty and will be dictated by subsequent critical events including age at first calving, duration of the postpartum interval for each successive calving, conception and pregnancy rate and ultimately manifested as length of intercalving intervals. Puberty in heifers is a consequence of the interactive effects of genetics and both pre and post-weaning nutrition. Early onset of puberty is essential to achieving the first main reproductive target for beef cow herds; first calving at two years of age. In calved heifers and mature cows, the onset of ovarian activity, postpartum is a key event dictating the calving interval. Again, this will be the product mainly of prepartum nutrition, manifested through BCS and the strength of the maternal bond between cow and calf, though there is increasing evidence of a modest genetic influence on this trait. Following the initiation of postpartum ovarian cyclicity, conception and subsequent pregnancy rate is generally a function of bull fertility in natural service herds and heat detection and timing of insemination in herds bred through AI. Cows and heifers should be maintained on a steady plane of nutrition during the breeding season, but the contribution of significant excesses or deficiencies of nutrients including protein and trace elements is likely to be minor where adequate pasture is available. While, increased efforts are being made internationally to genetically identify and select for more reproductively efficient beef cows, this is a more long-term strategy and will not replace the need for a high level of technical efficiency and management practice at farm level.
\end{abstract}

Conclusions Compact calving before turn out to pasture in spring is an essential component of pasture-based suckled beef production systems to ensure maximum herbage utilization and, hence, profitability. Achieving a highly concentrated period of calving in the spring requires early onset of puberty in replacement together with both high submission and high pregnancy rates in both heifers and cows, within a short period following the planned start of mating. In beef cows high submission rate in the first 6 weeks of the breeding period is highly dependent on cows having resumed oestrous cycles by 50 days post-calving. Indeed, compared with dairy cows, there is typically considerable variability in the duration of the postpartum anoestrous period in suckled beef cows with mean duration often extending to beyond 80 days even in cows of moderate to good BCS. While ovarian follicular development resumes early postpartum, the prolonged postpartum interval of suckled beef cows is due to the failure of successive dominant follicles to ovulate. This is a consequence of, in turn, to the inadequate frequency of LH pulses. While management practices such as restricted suckling and/or exposure to an intact male can cause an increase in the frequency of LH pulses and hasten the resumption of ovarian cyclicity in some cows, these approaches are often viewed as impractical and or labour intensive at farm level. Maximizing the proportion of cows that establish pregnancy within the first 42 days of the breeding season decreases the incidence of extended calving patterns. Later-calving cows with an extended postpartum anoestrous interval can disrupt the seasonal calving pattern and result in extended duration of calving. Furthermore, in beef cows nursing their calves, the expression of overt signs of oestrus are reduced, thus further increasing the difficulty of oestrous detection which is a prerequisite for using AI. Subfertility of bulls, although often a transient condition, can have devastating effects on achieving high herd fertility and requires on-going vigilance. While, increased efforts are being made internationally to genetically identify and select for more reproductively efficient beef cows, this is a more long-term strategy and will not replace the need for a high level of technical efficiency and management practice at farm level. 


\section{Parturition to resumption of ovarian cyclicity: comparative aspects of beef and dairy cows} M A Crowe $^{1^{*}}$, M G Diskin $^{2}$ and E J Williams ${ }^{1}$

${ }^{1}$ UCD Veterinary Sciences Centre, School of Veterinary Medicine, University College Dublin, Belfield, Ireland.

${ }^{2}$ Teagasc, Athenry, Co. Galway, Ireland.

Email: mark.crowe@ucd.ie

Implications Resumption of ovulation and oestrous cycles are required to facilitate rebreeding of postpartum cows. Beef cows have a prolonged interval to resumption of ovulation compared with dairy cows due to suckling and maternal bond inhibiting ovulation. The time to ovulation is limited by lack of the ovulation stimulus and not lack of follicle growth. In dairy cows the key inhibitors of resumption of ovulation include energy balance changes, body condition score at calving, dry matter intake and health disorders. First ovulation postpartum is generally silent (no expression of oestrous behavior) and is generally followed by a short cycle.

Abstract There is a variable anoestrous period following parturition in the cow. Follicular growth generally resumes within 7-10 days in the majority of cows associated with a transient follicle-stimulating hormone (FSH) rise that occurs within 3 to 5 days of parturition. Dairy cows that are not nutritionally stressed generally ovulate their first postpartum dominant follicle ( $\sim 15$ days), whereas beef suckler cows in good body condition normally have a mean of $3.2 \pm 0.2$ dominant follicles ( $\sim 30$ days) to first ovulation; and beef cows in poor body condition have a mean of $10.6 \pm 1.2$ dominant follicles ( 70-100 days) to first ovulation. The lack of ovulation of dominant follicles during the postpartum period is associated with infrequent luteinizing hormone (LH) pulses, with both maternal-offspring bonding and low body condition score at calving being implicated as the predominant causes of delayed resumption of cyclicity in nursed beef cows. In dairy cows the normal pattern of early resumption of ovulation may be delayed in high yielding Holstein type cows generally due to the effects of severe negative energy balance, dystocia, retained placental membranes and uterine infections. First ovulation, in both dairy and beef cows, is generally silent (i.e., no behavioural oestrus) and followed by a short inter-ovulatory interval $(>70 \%)$. The key to optimizing resumption of ovulation in both beef and dairy cows is appropriate pre-calving nutrition and management so that cows calve down in optimal body condition (body condition score; BCS; 2.75-3.0) with postpartum body condition loss restricted to $<0.5$ BCS units.

Summary and Conclusions Follicular growth generally resumes within 7-10 days postpartum in the majority of both dairy and beef cows and is associated with a transient FSH rise that occurs within 3 to 5 days of parturition. Delayed resumption of ovulation is invariably due to a GnRH mediated lack of LH pulse frequency whether it is due primarily to suckling inhibition in beef cows or metabolic related stressors in high yielding dairy cows. First ovulation in both dairy and beef cows is generally silent and followed by a short interovulatory interval (ovarian cycle). The key to optimizing resumption of ovulation in both beef and dairy cows is appropriate pre-calving nutrition and management so that cows calve down in optimal body condition (body condition score 2.75-3.0) with postpartum body condition loss restricted to $<0.5$ body condition score units. Genetic selection for increased milk yield in dairy cows increases metabolic and nutritional stressors, and in turn, may affect uterine health and / or uterine immune status that has consequences for clearance of uterine disease. 


\title{
Reproductive tract inflammatory disease in postpartum dairy cows
}

\author{
S J LeBlanc \\ Population Medicine, University of Guelph, Guelph, Ontario, Canada N1G 2W1 \\ Email: sleblanc@uoguelph.ca
}

Implications Reproductive disease in the first two months after calving is very common and spans severe clinical illness to unapparent yet substantial impairment of fertility. These conditions result from the interaction between infection and stimulus for inflammation and effective immune response and regulation of inflammation. Recent advances underline links among metabolism, inflammation, and health that were previously thought to be separate processes.

\begin{abstract}
Up to half of dairy cows are affected by at least one of metritis, purulent vaginal discharge, endometritis, or cervicitis in the postpartum period. These conditions result from inadequate immune response to bacterial infection (failure to clear pathogenic bacteria from the uterus) or persistent inflammation that impairs rather than enhances reproductive function. The degree of mobilisation of fat and how effectively it is used as a metabolic fuel is well recognized as a risk factor for metabolic and infectious disease. Release of non-esterified fatty acids has direct effects on liver and immune function but also produces pro-inflammatory cytokines (TNF $\alpha$ and IL-6) which contribute to systemic inflammation and to insulin resistance. Therefore, reproductive tract inflammatory disease may be a function of both local and systemic inflammatory stimuli and regulation as well as regulation of fat metabolism. Better understanding of variables associated with insulin resistance and inflammatory regulation in the liver and adipose tissue may lead to improvement of reproductive tract health. This paper reviews factors that may contribute to postpartum reproductive tract inflammatory diseases in dairy cows and their inter-relationships, impacts, and treatment.
\end{abstract}

Conclusions The balance between bacterial infection of the uterus in the weeks after calving and the effectiveness of the immune and inflammatory response to it determine the incidence of reproductive tract diseases. Understanding of links among metabolism and inflammation may lead to better approaches to prevention of reproductive disease through nutrition and management and more targeted therapies. 


\section{Maternal-embryo interaction leading up to the peri-implantation period of pregnancy in cattle}

P Lonergan ${ }^{1}$, N Forde

School of Agriculture and Food Science, Belfield, Dublin 4, Ireland

Email:pat.lonergan@ucd.ie

Implications Embryo mortality is a serious issue in cattle, particularly dairy cattle, and can have a significant impact on profitability, especially in a pasture-based system where there is a narrow window of opportunity in which to get cows back incalf after calving. Interaction between the developing embryo and the maternal reproductive tract is essential for the establishment of pregnancy and asynchrony between these two players can result in compromised embryo development and or reduced uterine receptivity.

Abstract Early embryo development following fertilization occurs in the oviduct. However, despite being the site of fertilization in cattle, it is possible to by-pass the oviduct by producing embryos in vitro and/or by transferring blastocysts recovered from one female into the uterus of another. While there is substantial evidence for the oviduct having an influence on the quality of the developing embryo, manifested in altered morphology, gene expression and cryotolerance, evidence for a two-way dialogue is weak. In contrast, successful growth and development of the post-hatching blastocyst and pregnancy establishment are a result of the two-way interaction between a competent embryo and a receptive uterine environment. Progesterone (P4) plays a key role in reproductive events associated with establishment and maintenance of pregnancy through its action on the uterine endometrium. Elevated concentrations of circulating P4 in the immediate postconception period have been associated with an advancement of conceptus elongation, an increase in interferon-tau production and, in some studies, higher pregnancy rates in cattle. This review summarizes current knowledge on the communication between the developing embryo and the maternal reproductive tract.

Conclusion In conclusion, there is significant evidence that exposure of the embryo to the oviduct benefits the embryo but with little evidence that the embryo itself elicits an effect on the oviduct. There is considerable evidence that changes to the endometrium (and subsequent histotroph) modulate the environment to which the embryo is exposed and affect the ability of the uterus to drive conceptus elongation. In addition to maternal to embryo communication, there is reciprocal cross-talk from the conceptus to the endometrium; this is mainly mediated by IFNT but there is new evidence that the conceptus modulates the endometrium by secreting molecules other than IFNT, including prostaglandins. 


\title{
Physiological and practical effects of progesterone on reproduction in dairy cattle
}

\author{
M C Wiltbank ${ }^{1}$, A H Souza ${ }^{1,2}$, P D Carvalho ${ }^{1}$, A P Cunha ${ }^{1}$, J O Giordano ${ }^{1,3}$, P M Fricke ${ }^{1}$, G M Baez ${ }^{1}$, M G Diskin ${ }^{4}$
}

${ }^{1}$ Department of Dairy Science, University of Wisconsin-Madison, Wisconsin USA

${ }^{2}$ Current Address: University of California Cooperative Extension, California USA

${ }^{3}$ Current Address: Department of Animal Science, Cornell University, New York USA

${ }^{4}$ Animal and Grassland Research and Innovation Centre, Teagasc Ireland

Email:Wiltbank@wisc.edu

Implications This reviews the effects of circulating progesterone (P4) on dairy cattle reproduction. Various methods to elevate P4 during growth of the preovulatory follicular wave have been shown to increase pregnancies/AI and reduce double ovulation, providing methods to improve fertility and reduce twinning rate in lactating dairy cattle. Conversely, very low concentrations of P4 near AI are needed to optimize fertility. Finally, elevations of P4 after AI can impact embryonic development and also may elevate fertility. Thus, innovative strategies to optimize circulating P4 concentrations during selected reproductive periods enhance our management tools for improving reproductive efficiency of lactating dairy cows.

\begin{abstract}
The discovery of progesterone (P4) and elucidation of the mechanisms of P4 action have an important place in the history of endocrinology and reproduction. Circulating P4 concentration is determined by a balance between P4 production, primarily by the corpus luteum (CL), and P4 metabolism, primarily by the liver. The volume of luteal tissue and number and function of large luteal cells are primary factors determining P4 production. Rate of P4 metabolism is generally determined by liver blood flow and can be of critical importance in determining circulating P4 concentrations, particularly in dairy cattle. During timed AI protocols, elevations in P4 are achieved by increasing number of CL by creating accessory CL or by supplementation with exogenous P4. Dietary manipulations can also alter circulating P4, although practical methods to apply these techniques have not yet been reported. Elevating P4 prior to the timed AI generally decreases double ovulation and increases fertility to the timed AI. Near the time of AI, slight elevations in circulating P4, possibly due to inadequate luteal regression, can dramatically reduce fertility. After AI, circulating P4 is critical for embryo growth and establishment and maintenance of pregnancy. Many studies have attempted to improve fertility by elevating P4 after timed AI. Our recent meta-analysis and manipulative study indicated small fertility benefits (3-3.5\%) mostly in primiparous cows. Thus, previous research has provided substantial insight into mechanisms regulating circulating P4 concentrations and actions. Understanding this prior research can focus future research on P4 manipulation to improve reproductive success.
\end{abstract}

Conclusions This manuscript has attempted to describe the underlying physiology that produces the changes in circulating P4 in lactating dairy cows and the potential reproductive challenges associated with sub-optimal P4 concentrations. Metabolism of P4 appears to be the primary cause of lowered P4 in lactating dairy cows, although increases in P4 production by the CL may be important in higher fertility genotypes of dairy cows. This manuscript reviewed the scientific literature on P4 and fertility with clear evidence for effects of P4 at all three time periods that were analyzed. Prior to AI, there were very dramatic effects observed when the P4 concentrations before AI were compared to subsequent fertility or when manipulative studies were performed to increase P4 before AI. Improvements of more than 10\% in P/AI were observed by increasing P4 concentrations. In addition, insufficient P4 at this time may, at least partially, underlie the high double ovulation rate as well as the lowered fertility that is characteristic of high-producing dairy cows. Near the time of AI, it is critical that P4 concentrations reach a nadir concentration. Even small increases in P4 near the time of AI were associated with dramatic reductions in fertility, either in cows bred to natural estrus or after timed AI protocols. Following AI, there are dramatic effects of increasing P4 on embryo elongation. In addition, there have been relationships found between P4 concentrations after AI and subsequent fertility when linear regression analyses were performed. However, manipulative studies have not provided consistent or dramatic effects of P4 supplementation on fertility in most studies that have evaluated large numbers of cows. Thus, although substantial research has investigated the role of P4 on fertility in lactating dairy cows for more than 6 decades, it seems clear that future research is needed to fully understand the physiology that underlies previous research observations and to unlock the practical improvements in fertility that are expected by consistent management of P4 concentrations in lactating dairy cows. 


\section{Endocrine and metabolic mechanisms linking postpartum glucose with early embryonic and foetal development in dairy cows}

M C Lucy ${ }^{1}$, S T Butler ${ }^{2}$, and H A Garverick ${ }^{1}$

${ }^{1}$ Division of Animal Sciences, University of Missouri, Columbia, Missouri, USA

${ }^{2}$ Animal \& Grasslands Research and Innovation Centre, Teagasc Moorepark, Fermoy Co Cork, Ireland

Email: lucym@missouri.edu

Implications Competing processes of milk production and reproduction can be at odds in dairy cows, particularly if the unique metabolic processes that typify early lactation become imbalanced. A potential end result is that cows do not become pregnant during the breeding period. The need to replace infertile cows adds to the cost of production. Understanding basic mechanisms that link the first 60 days of lactation with the subsequent success or failure of establishing pregnancy during the breeding period, therefore, is an important area of research for the dairy industry.

Abstract Milk and milk solids production per cow is increasing annually in dairy systems. Peak milk production is in early lactation when the uterus and ovary are recovering from the previous pregnancy. The competing processes of milk production and restoration of reproductive function can be at odds, particularly if unique homeorhetic mechanisms that typify early lactation become imbalanced and cows experience metabolic disease. Homeorhesis leads to an increase in the synthesis of glucose that is irreversibly lost to milk lactose. Irreversible loss of glucose during lactation can invoke an endocrine and metabolic state that impinges upon postpartum uterine health, oestrous cyclicity and subsequent establishment of pregnancy. The first 30 days postpartum may be most-critical in terms of the impact that metabolites and metabolic hormones have on reproduction. Depressed immune function caused in part by the postpartum metabolic profile leads to a failure in uterine involution and uterine disease. Oestrous cyclicity (interval to first ovulation and subsequent periodicity) is affected by the same hormones and metabolites that control postpartum immune function. Slower growth of the embryo or foetus perhaps explained by the unique metabolic profile during lactation may predispose cows to pregnancy loss. Understanding homeorhetic mechanisms that involve glucose and collectively affect postpartum uterine health, oestrous cyclicity and the establishment of pregnancy should lead to methods to improve postpartum fertility in dairy cows.

Conclusions The endocrine and metabolic environment of lactating cows affects the capacity of cows to become pregnant postpartum. There is ample evidence that the hormones that coordinate homeorhetic adjustments that support lactation can act on the uterus and ovary before and during the breeding period. In addition to the hormonal environment, the metabolic environment created by lactation that includes low blood glucose and elevated NEFA and BHBA impinges upon the ovary as well as the immune system that plays a critical role in restoring uterine health in postpartum cows. Specific mechanisms through which the metabolic environment of early lactation deposits a lasting imprint on uterine and ovarian function is less clear. Also less clear are the mechanisms that link lactation to a predisposition for pregnancy loss in lactating cows. The slow rate of embryonic or foetal growth in lactating cows with low blood progesterone and low blood concentrations of glucose may be an important mechanism explaining pregnancy loss. 


\title{
Heifer fertility and carry over consequences for life time production in dairy and beef cattle
}

D C Wathes ${ }^{1}$, G E Pollott ${ }^{1}$, K F Johnson ${ }^{1}, \mathrm{H}$ Richardson ${ }^{2}$ and J S Cooke ${ }^{3}$

${ }^{1}$ Department of Production and Population Health, Royal Veterinary College, Hawkshead Lane, North Mymms, Hatfield, Herts, AL9 7TA, UK.

${ }^{2}$ Centre for Dairy Information, Scotsbridge House, Scots Hill, Rickmansworth, Herts, WD3 3BB, UK.

${ }^{3}$ Volac, Orwell, Royston, Herts, SG8 5QX, UK.

Email:dcwathes@rvc.ac.uk

Implications Cattle do not calve until around 2 years of age, so the costs of their early development need to be repaid from later sales of milk and meat. Economic calculations show that calving at 23-25 months is optimal. To achieve this heifers must conceive by 15 months. Inadequate growth rates due to poor nutrition and calf disease can compromise development and reduce future production potential.

\begin{abstract}
The rearing period has a key influence on the later performance of cattle, affecting future fertility and longevity. Producers usually aim to breed replacement heifers by 15 months to calve at 24 months. An age at first calving (AFC) close to 2 years (23-25 months) is optimum for economic performance as it minimises the non-productive period and maintains a seasonal calving pattern. This is rarely achieved in either dairy or beef herds, with average AFC for dairy herds usually between 26-30 months. Maintaining a low AFC requires good heifer management with adequate growth to ensure an appropriate body weight (BW) and frame size at calving. Puberty should occur at least 6 weeks before the target breeding age to enable animals to undergo oestrous cycles before mating. Cattle reach puberty at a fairly consistent, but breeddependent, proportion of mature BW. Heifer fertility is a critical component of AFC. In US Holsteins the conception rate peaked at 57\% at $15-16$ months, declining in older heifers. Wide variations in growth rates on the same farm often lead to some animals having delayed first breeding and/or conception. Oestrous synchronisation regimes and sexed semen can both be used but unless heifers have been previously well-managed the success rates may be unacceptably low. Altering the nutritional input above or below those needed for maintenance at any stage from birth to first calving clearly alters the average daily gain in weight (ADG). In general an ADG of around $0.75 \mathrm{~kg} / \mathrm{d}$ seems optimal for dairy heifers, with lower rates delaying puberty and AFC. There is some scope to vary ADG at different ages providing animals reach an adequate size by calving. Major periods of nutritional deficiency and/or severe calfhood disease will, however, compromise development with long term adverse consequences. Infectious disease can also cause pregnancy loss/abortion. First lactation milk yield may be slightly lower in younger calving cows but lifetime production is higher as such animals usually have good fertility and survive longer. There is now extensive evidence that as long as the AFC is $>23$ months then future performance is not adversely influenced. On the other hand, delayed first calving $>30$ months is associated with poor survival. Underfeeding of young heifers reduces their milk production potential and is a greater problem than overfeeding. Farmers are more likely to meet the optimum AFC target of 23-25 months if they monitor growth rates and adjust feed accordingly.
\end{abstract}

Conclusions The breeding performance of heifers is fundamental for farm profitability. Adequate numbers of replacement animals must be reared which are healthy, calve in a timely fashion and go on to produce calves and milk on a regular basis. This requires good fertility to promote longevity. In order for animals to calve at around 2 years of age they must have been fed sufficiently at all stages not to compromise their growth and they need to remain healthy. Concerns have been raised that high growth rates and calving at 2 years may predispose to calving difficulties and compromise long term milk production. The majority of evidence, however, supports the view that an AFC of 23-25 months is optimal economically and does not have any adverse consequences as long as the heifers are of an adequate body weight and stature. On the other hand, excessively high growth rates may be detrimental to fertility and are both unnecessary and uneconomic. Late calving cows are also more likely to be overweight and this also causes problems in the first lactation. Current evidence suggests that under nutrition of young dairy heifers is a widespread problem which should be addressed. Improved monitoring of growth at regular intervals on both beef and dairy units would aid farmers to optimise their heifer management. Economic studies are needed which model the effects of different levels of input to replacement dairy and beef heifers on their subsequent lifetime productivity. These should help to convince farmers that costs associated with putting sufficient resources into rearing well grown and healthy youngstock will be recouped by producing better cows. 


\title{
Genetics and genomics of reproductive performance in dairy and beef cattle
}

\author{
D P Berry ${ }^{1}$, E Wall ${ }^{2}$, and J E Pryce
}

${ }_{1}^{1}$ Animal \& Grassland Research and Innovation Centre, Teagasc, Moorepark, Co. Cork, Ireland.

${ }^{2}$ Animal and Veterinary Sciences, SRUC, Kings Buildings, West Mains Road, EH9 3JG, United Kingdom

${ }^{3}$ Dept. of Environment and Primary Industries \& Dairy Futures CRC, Agribio, 5 Ring Road, La Trobe University, Bundoora, 3083, Australia

Email: donagh.berry@teagasc.ie

Implications Excellent reproductive performance in both males and females is fundamental to profitable dairy and beef production systems. Genetics is known to contribute to variation in cattle performance traits including milk and meat production. In this review we summarise the contribution of genetic differences to phenotypic performance, how the genetics of reproductive performance is correlated with the genetics of other performance traits. Particular attention is given to gaps in knowledge as well as potentially useful technologies in the near future.

\begin{abstract}
Excellent reproductive performance in both males and females is fundamental to profitable dairy and beef production systems. In this review we undertook a meta-analysis of genetic parameters for female reproductive performance across 55 dairy studies or populations and 12 beef studies or populations as well as across 28 different studies or populations for male reproductive performance. A plethora of reproductive phenotypes exist in dairy and beef cattle and a meta-analysis of the literature suggests that most of the female reproductive traits in dairy and beef cattle tend to be lowly heritable ( 0.03 to 0.04$)$. Reproductive-related phenotypes in male animals (e.g., semen quality) tend to be more heritable than female reproductive phenotypes with mean heritability estimates of between 0.05 and 0.22 for semen-related traits with the exception of scrotal circumference $(0.42)$ and field non-return rate $(0.001)$. The low heritability of reproductive traits females, in particular, does not however imply that genetic selection cannot alter phenotypic performance as evidenced by the decline until recently in dairy cow reproductive performance attributable in part to aggressive selection for increased milk production. Moreover, the antagonistic genetic correlations among reproductive traits and both milk (dairy cattle) and meat (beef cattle) yield is not unity thereby implying that simultaneous genetic selection for both increased (milk and meat) yield and reproductive performance is indeed possible. The required emphasis on reproductive traits within a breeding goal to halt deterioration will vary based on the underlying assumptions and is discussed using examples for Ireland, the UK and Australia as well as quantifying the impact on genetic gain for milk production. Advancements in genomic technologies can aid in increasing the accuracy of selection for especially reproductive traits and thus genetic gain. Elucidation of the underlying genomic mechanisms for reproduction could also aid in resolving genetic antagonisms. Past breeding programs have contributed to the deterioration in reproductive performance of dairy and beef cattle. The tools now exist, however, to reverse the genetic trends in reproductive performance underlying the observed phenotypic trends.
\end{abstract}

Conclusions The heritability of most traditional measures of male and female reproductive performance in dairy and beef cattle is low. However, this does not imply that genetic gain in reproductive performance is not possible as evidenced by its deterioration in past decades in most Holstein populations. Therefore, genetic selection for improvements in reproductive performance is indeed possible. Since the genetic correlations with output traits (i.e., milk production and growth rate) are not unity, genetic gains in reproductive performance does not imply a reduction in genetic merit for output traits. Genomic information is now included in most developed national dairy cattle breeding programs and research into implementation in beef breeding programs is actively underway. Retrospective analysis to-date signifies clear advantages in genetic gain through exploitation of genomic information. Moreover, advancements in genomics research can further improve the rate of genetic gain for all traits affecting profitability, including reproductive performance. 


\title{
Crossbreeding - Implications for dairy cow fertility
}

\section{F Buckley ${ }^{1}$, N Lopez-Villalobos ${ }^{2}$ and B J Heins ${ }^{3}$}

${ }^{1}$ Animal and Bioscience Research Department, Animal and Grassland Research and Innovation Centre, Teagasc,

Moorepark, Fermoy, Co. Cork, Ireland.

${ }^{2}$ Institute of Veterinary, Animal and Biomedical Sciences, Massey University, Palmerston North, New Zealand

${ }^{3}$ Department of Animal Science, University of Minnesota, St. Paul 55108, USA

Email: frank.buckley@teagasc.ie

Implications Crossbreeding of dairy cattle has become a topic of some considerable interest in response to concerns by dairy producers about declining fertility, health and survival with Holstein cows. By crossbreeding, producers aim to exploit favourable characteristics of 'alternative' breeds, remove the negative effects associated with inbreeding, and capitalise on a phenomenon known as heterosis. Recent research results clearly illustrate that the net effect is favourable animal performance, using a range of modern breeds, and within the context of both grass-based and high input confinement production environments. Economic analyses indicate profitable performance due to lower replacement cost and higher herd productivity.

\begin{abstract}
In pasture-based seasonal calving systems failure to become pregnant during the breeding season results in important economic losses, as maximum profit is attained by minimizing costs and increasing the proportion of grazed grass in the diet of the lactating dairy cow. In the United States dairy producers with Holsteins have observed decreased cow fertility and survival. Crossbreeding exploits favourable characteristics of a second breed, removes the negative effects of inbreeding, and capitalises on heterosis; the net effect generally being improved fertility and survival. Research conducted in Ireland, by Teagasc, during the early 2000's concluded that of three 'alternative' breeds, Montbeliarde, Normande and Norwegian Red, the latter was most suited to seasonal grass-based production, due to favourable performance including fertility. A follow up study found a clear fertility advantage with Norwegian Red $\times$ Holstein-Friesian compared with Holstein-Friesian, e.g., proportion pregnant to first service $(+0.08)$ and in-calf after 6 weeks breeding $(+0.11)$. A further study found significant fertility benefits with Jersey crossbreds, e.g., pregnant to first service $(+0.21)$ and in-calf after 6 weeks breeding (+0.19). Similar performance with Jersey crossbred cows was observed at the Agri-Food and Biosciences Institute, Hillsborough, Northern Ireland. In New Zealand crossbred dairy cattle are now the most prevalent. There, the crossbred population (primarily Jersey $\times$ Friesian) is achieving similar rates of genetic gain for farm profit as the purebred populations, but creating additional gain derived by the economic heterosis. In the United States, analysis of commercial data from California showed higher first-service conception rates for Scandinavian Red $\times$ Holstein $(+6 \%)$ and Montbeliarde $\times$ Holstein $(+10 \%)$ compared with Holstein $(23 \%)$. They also exhibited fewer days open and greater survival. At Penn State University Brown Swiss $\times$ Holstein cows had 17 fewer days open than Holstein cows during first lactation, and while numerically fewer in second (12 days) and third lactation ( 6 days), these values were not significantly different to the Holstein. At the University of Minnesota, Montbeliarde $\times$ Holstein and Montbeliarde $\times$ Jersey/Holstein crossbred cows had $21 \%$ higher first service conception rate, e.g., 41 fewer days open, and $12 \%$ higher in-calf rates compared with the Holstein cows. They also had greater survival to second $(+13 \%)$, third $(+24 \%)$, fourth $(+25 \%)$, and fifth $(+17 \%)$ lactation. In summary, the literature clearly illustrates favourable animal performance benefits from crossbreeding, using a range of modern breeds, and within the context of both grass-based and high input confinement production environments. Economic analyses, generally indicates profitable performance due to lower replacement cost and higher herd productivity.
\end{abstract}

Concluding Remarks Crossbreeding should be regarded as a mating system that complements genetic improvement within breeds. Continuous use of progeny-tested and highly-ranked artificial insemination bulls is critical to genetic improvement regardless of mating system. Heterosis is a bonus that dairy producers can expect in addition to the positive effects of individual genes obtained by using superior artificial insemination bulls within breed. The bonus from heterosis is in the range of $2 \%$ to $5 \%$ for production and at least $10 \%$ for mortality, fertility, health, and survival, and heterosis comes on top of the average genetic level of the two parent breeds. Some dairy producers might need to get beyond the notion that level of milk production is the only measure of profitability of dairy cows.

The findings from the research presented, clearly illustrates favourable animal performance benefits from crossbreeding, using a range of modern breeds, and within the context of both low cost (grass-based) and high input confinement production environments. Crossbreeding is clearly a very powerful means to improve the fertility/longevity performance of commercial dairy herds and in so doing improve their long term profitability and sustainability. A study of Kargo et al. (2012) supports the notion that crossbreeding is a system that should be considered valuable for all management levels. Evidence from New Zealand would concur that despite increased additive genetic gain within the main purebred populations it is the crossbred cows that continue to generate greatest profit. In New Zealand, the proportion of dairy cows categorised as crossbred has reached an all time high. For the first time over $40 \%$ of dairy cows and $50 \%$ of replacement females are crossbred, making crossbreds officially the dairy cow of choice (New Zealand dairy statistics, 2012-13). Elsewhere, a fear of the unknown, or perhaps a conservative bias or mindset, would appear to be restricting the growth of what might pragmatically be recognised as a prudent use of available genetic resources to maximise profitable dairy production. In Ireland, crossbreds currently represent approximately $8 \%$ of dairy births. In the United States, the percentage of the national herd that was crossbred increased from 0.6 for cows born in 1990 to 3.0\% for cows born in 2005 (Powell et 
al., 2008). In 2012, 8.4\% of the dairy cattle population in the United States was crossbred in Dairy Herd Improvement herds (AIPL, 2012)

In summary, despite the very positive and consistent research findings, as stated by McAllister (2012), the rather limited but current interest in crossbreeding in commercial US dairy industry is an interesting development. The sentiment is telling, and equally valid for most countries where positive results pertaining to crossbreeding in the dairy herd has been observed.

\section{References}

Kargo M, Madsen P and Norberg E 2012. Journal of Dairy Science 95, 925-928.

McAllister AJ 2002. Journal of Dairy Science 85, 2352-2357.

Powell RL, Norman HD and Hutchison JL 2008.. Journal of Dairy Science 91, (E-Suppl. 1):7(abstract T17). 


\title{
Expression and detection of oestrus in dairy cows: The role of new technologies
}

\section{P M Fricke, P D Carvalho, J O Giordano ${ }^{1}$, A Valenza ${ }^{2}$, G Lopes $\mathrm{Jr}^{3}$, M C Amundson}

Department of Dairy Science, University of Wisconsin, Madison, WI 53706-1205, USA

${ }^{1}$ Current address: Department of Animal Science, Cornell University, Ithaca, NY 14853, USA.

${ }^{2}$ Current address: CEVA Sante Animale, 10 avenue la Ballastière 33500, Libourne, France.

${ }^{3}$ Current address: Accelerated Genetics / World Wide Sires, Baraboo, WI, 53913, USA

\section{Email: pmfricke@wisc.edu}

Implications A new generation of electronic systems that continuously monitor physical activity of dairy cows to predict timing of artificial insemination have been developed. In two experiments on a large commercial dairy in the U.S., an activity monitoring system detected only $70 \%$ of lactating Holstein cows with increased activity with the remainder of cows being anovular or ovulating in the absence of activity. A combined approach in which AI based on activity is followed by submission of cows not detected with activity to timed AI after synchronization of ovulation may be an effective strategy for submission of cows to first AI.

\begin{abstract}
Despite the widespread adoption of hormonal synchronization protocols that allow for timed artificial insemination (AI), detection of oestrus plays an important role in the reproductive management program on most dairies in the U.S. Increased physical activity is a secondary sign of oestrus in dairy cattle, and a new generation of electronic systems that continuously monitor physical activity to predict timing of AI have been developed and marketed to the dairy industry. A variety of management and physiologic challenges inhibit detection of behavioral oestrus on farms, but the prevalence of anouvular cows near the end of the voluntary waiting period is particularly problematic. Only $70 \%$ of lactating Holstein cows were detected in oestrus when using an activity monitoring system, with the remaining $20 \%$ of cows classified as anovular and $10 \%$ ovulating without showing signs of activity. Mean time of AI in relation to ovulation based on the activity monitoring system was acceptable for most of the cows with increased activity; however, variability in the duration of oestrus and timing of $\mathrm{AI}$ in relation to ovulation could result in poor pregnancy outcomes in some cows. Use of a Presynch-Ovsynch protocol for submission of cows for first AI has been widely adopted by dairies in the U.S., and a combined approach in which AI based on activity is followed by submission of cows not detected with activity to timed AI after synchronization of ovulation may be an effective strategy for submission of cows to first AI. Based on a field trial on a large commercial dairy in the U.S., the activity monitoring system detected $70 \%$ of cows with increased activity after the second $\mathrm{PGF}_{2 \alpha}$ injection of a Presynch-Ovsynch protocol; however, cows inseminated to increased activity had fewer pregnancies per artificial insemination (P/AI) compared to cows with increased activity after the second $\mathrm{PGF}_{2 \alpha}$ injection that received timed AI after completing the Presynch-Ovsynch protocol. Based on an economic model comparing reproductive management programs with varying levels of AI to oestrus versus timed AI, the rate of oestrous detection and the P/AI to inseminations based on AI to detected oestrus versus timed AI affected the decision to inseminate based on activity versus timed AI. In conclusion, an activity monitoring system detected increased activity in about $70 \%$ of lactating Holstein cows on a large commercial dairy in the U.S.; however, synchronization of ovulation and timed AI was beneficial to inseminate cows not detected with increased activity by the activity monitoring system.
\end{abstract}

Summary and Conclusions Based on two experiments assessing an activity monitoring system on a large commercial dairy farm in the U.S. (Valenza et al., 2012; Fricke et al., 2012) only about 70\% of cows were inseminated based on the activity monitoring system. These data underscore the importance of implementing a comprehensive reproductive management program for identification and treatment of cows that would otherwise not be inseminated. The mean time of $\mathrm{AI}$ in relation to ovulation was acceptable for most of the cows detected in oestrus; however, variability in the duration of oestrus and timing of AI in relation to ovulation could lead to poor fertility in some cows (Valenza et al., 2012). Although use of an activity monitoring system to inseminate cows based on activity reduced days to first AI, cows receiving $100 \%$ timed AI after completing a Presynch-Ovsynch protocol had more P/AI (Fricke et al., 2012) . The trade-off between AI service rate and P/AI was reflected by an economic analysis in which varying levels of oestrous detection and three levels of P/AI were compared (Giordano et al., 2012). When P/AI to detected oestrus in a herd is poor, allowing cows to complete the Presynch-Ovsynch protocol and receive a timed AI would be a preferred strategy; conversely, whenever P/AI to AI after a detected oestrus is $30 \%$ or $35 \%$ there was always an economic advantage to inseminating cows detected in oestrus across all levels of oestrous detection.

We conclude that although a variety of strategies can be used to submit cows for first and subsequent AI, synchronization of ovulation and timed AI is a beneficial strategy to inseminate cows not detected by the activity monitoring system. Clearly much effort has been invested in developing systems for detecting oestrus in dairy cows. Refinement of existing technologies as well as development of new technologies for detection of oestrus will likely continue in the future. It will indeed be interesting to see how physiological changes in dairy cows due to high milk production affect the ability of cows to express oestrus as well as affect the ability of technologies to accurately detect oestrus in dairy cows.

References Fricke PM, Valenza A, Giordano JO, Amundson MC and Lopes Jr G 2012. Journal of Dairy Science 95(Supplement 2), 74 (Abstract).

Giordano JO, Kalantari AS, Fricke PM, Wiltbank MC and Cabrera VE 2012. Journal of Dairy Science 95, 5442-5460.

Valenza A, Giordano JO, Lopes Jr G, Vincenti L, Amundson MC and Fricke PM 2012. Journal of Dairy Science 95, 71157127. 


\author{
Synchronization of ovulation and fixed-time artificial insemination in beef cattle \\ $\mathrm{G} \mathrm{A} \mathrm{Bó}^{125}$ and P S Baruselli ${ }^{3}$ \\ ${ }^{1}$ Instituto de Reproducción Animal Córdoba (IRAC), Zona Rural General Paz, 5145, Córdoba, Argentina. \\ ${ }^{2}$ Instituto A.P. de Ciencias Básicas y Aplicadas, Carrera de Medicina Veterinaria, Universidad Nacional de Villa María, \\ Córdoba, Argentina. \\ ${ }^{3}$ Departamento de Reprodução Animal, FMVZ-USP, CEP 05508-000 São Paulo, Brazil. \\ Email:gabrielbo@iracbiogen.com.ar.
}

Implications The use of protocols that control follicular development and ovulation, commonly known as fixed-time AI protocols, has the advantage of being able to apply assisted reproductive technologies without the need for detecting oestrus. These treatments have been shown to be practical and easy to perform by the farm staff, and more importantly, they do not depend on the accuracy in oestrus detection. These treatments have been proven to be efficient, with repeatable results and are the best way of introducing the best genetics in a herd through artificial insemination.

\begin{abstract}
The main objective of the implementation of Artificial Insemination (AI) in cattle is to produce a sustained genetic progress in the herd. Although AI is an old reproductive biotechnology, its widespread implementation is very recent and is mainly due to the use of protocols that allows AI without oestrus detection, commonly called fixed-time artificial insemination (FTAI). The development of FTAI protocols also allowed the application of AI in larger, extensively managed, herds and especially in suckled cows instead of restricting the breeding programs to the heifers. Fixed-time AI treatments are widely used in South America, with about 3,000,000 cows inseminated in the last season in Argentina and about 8,000,000 in Brazil. The objective of this review is to present and describe the various treatments available and some of the factors that may affect pregnancy rates in beef cattle.
\end{abstract}

Summary and conclusions The use of protocols that control follicular development and ovulation has the advantage of being able to apply assisted reproductive technologies without the need for detecting oestrus. These treatments have been shown to be practical and easy to perform by the farm staff, and more importantly, they do not depend on the accuracy in oestrus detection. Treatments with GnRH or oestradiol and progestin-releasing devices have provided for FTAI in beef cattle, and the addition of eCG has been especially useful in increasing pregnancy rates in cows experiencing postpartum anoestrus. Shorter ovulation synchronization treatments that provide for a longer proestrus are an interesting new alternative for FTAI; however, more studies are needed to determine if these treatments increase P/AI compared to the traditional EB plus progestin treatments currently used by most practitioners in South-America. Finally, these treatments have been proven to be efficient, with repeatable results and are the best way of introducing the best genetics in a herd through artificial insemination. 


\title{
Synchronization of ovulation for management of reproduction in dairy cows
}

\author{
R S Bisinotto and J EP Santos \\ Department of Animal Sciences, University of Florida, 2250 Shealy Drive, Gainesville, FL, 32611 USA \\ Email: Jepsantos@ufl.edu
}

Implications Consolidation of the dairy industry with constant increase in herd size has generated the need for systematic programs to manage reproduction. The advent of synchronization of ovulation protocols that secure artificial insemination (AI) at a pre-established time with adequate fertility has become an integral component of breeding management in multiple production systems. The better understanding of ovarian biology of the dairy cows with improved control of follicle growth and luteal lifespan has resulted in refinement of these programs in the last two decades providing unique opportunities to manipulate follicle development, improve oocyte quality and enhance embryo survival.

\begin{abstract}
Important developments have occurred in the last two decades, since the advent of the Ovsynch protocol, on the understanding and use of synchronization programs for management of reproduction in dairy herds. This better understanding of estrous cycle control associated with suboptimal reproductive performance in dairy herds has lead dairy producers to quickly adopt timed artificial insemination (AI) protocols. Recent surveys have documented that protocols for fixed time AI have become integral components of management of reproduction in high-producing herds. Furthermore, timed AI protocols have also demonstrated benefits in grazing farms because of the inability to efficiently detect estrus in dairy cows. In general, successful use of the Ovsynch protocol requires some fundamental physiological principles to be respected, including: induction of ovulation to synchronize follicle growth in the first two days of the program such that a young antral follicle is recruited; maintenance of high concentrations of progesterone during the development of the ovulatory follicle, but also effectively lyse the corpus luteum (CL) to result in very low concentration of progesterone at AI; and having a healthy pre-ovulatory follicle of moderate diameter that is highly estrogenic and responsive to gonadotropins to synchronously ovulate 12 to 18 hours after insemination. Current methods to improve these responses during the Ovsynch protocol are still not optimal and future developments will likely require new technologies for hormone formulation and delivery such that additional interventions are minimized to maintain acceptance by producers.
\end{abstract}

Conclusion Manipulation of the estrous cycle to improve insemination rates continues to be an area of major interest and of active research. Timed AI has become an integral component of reproductive management in many dairy herds because of challenges with detection of estrus either because of poor expression by cows or the inability of systems in place to identify these animals in estrus. In spite of decades of research and numerous studies, timed AI protocols based on GnRH and $\mathrm{PGF}_{2 \alpha}$ have limitations on the ability to synchronize the emergence of follicle growth, induce complete CL regression, and have a synchronized ovulation at a predictable time to optimize fertility. In most cases, less than $60 \%$ of the cows experience all these pre-requisites for high fertility. Advantage should be taken to optimize pregnancy at the first postpartum insemination because of the ability to presynchronize the estrous cycle and improve cow fertility with timed AI programs. Unfortunately, until new technologies for hormone formulation and delivery become available, improvements in synchrony of follicle growth, luteal regression, and ovulation will require additional interventions that might make these programs less practical or acceptable by producers. It is clear that current and future research need to focus on target groups of cows, such as anovular cows and cows with less than optimal follicle competence and steroid concentrations to maximize response to treatments. Nevertheless, additional fertility responses to synchronization programs will have to move beyond hormonal manipulations only and will require more holistic approach to reproduction, integrating improvements in peripartum cow health and nutrition. 


\section{Update on sexed semen technology in cattle \\ G E Seidel, Jr. \\ Animal Reproduction and Biotechnology Laboratory, Colorado State University, Fort Collins, CO 80523-1683 USA \\ george.seidel@colostate.edu}

Implications Over the next few years, it is unlikely that any fundamentally new technology will become available for sexing sperm commercially. However, incremental improvements in flow cytometer/cell sorting procedures almost certainly will result in faster speeds of sexing sperm and improved fertility. However, fertility of sexed semen is unlikely to exceed $90 \%$ of unsexed semen for most situations for several years, so profitable applications for cattle will be limited. Longer term prospects are encouraging; since sperm are evaluated one at a time, some defective sperm are discarded during sexing, and this may become more sophisticated, increasing fertility.

Abstract The technology in current use for sexing sperm represents remarkable feats of engineering. These flow cytometer/cell sorters can make over 30000 consecutive evaluations of individual sperm each second for each nozzle and sort the sperm into three containers: X-sperm, Y-sperm, and unsexable plus dead sperm. Even at these speeds it is not economical to package sperm at standard numbers per inseminate. However, with excellent management, pregnancy rates in cattle with 2 million sexed sperm per insemination dose are about $80 \%$ of those with conventional semen at normal sperm doses. This lowered fertility, in part due to damage to sperm during sorting, plus the extra cost of sexed semen limits the applications that are economically feasible. Even so, on the order of 2 million doses of bovine semen are sexed annually in the United States. The main application is for dairy heifers to have heifer calves, either for herd expansion or for sale as replacements, often for eventual export. Breeders of purebred cattle often use sexed semen for specific matings; thawing and then sexing frozen semen and immediately using the few resulting sexed sperm for IVF is done with increasing frequency. Beef cattle producers are starting to use sexed semen to produce crossbred female replacements. Proprietary improvements in sperm sexing procedures, implemented in 2013, are claimed to improve fertility between 4 and 6 percentage points, or about $10 \%$.

Prospects for new methods of sexing sperm Sex is the most important genetic trait, and eventually it is likely that procedures will be developed that do not require evaluation of each individual sperm in series. However, nature has imposed a series of formidable barriers to prevent the genotype (including sex chromosome composition) of sperm from affecting phenotype of sperm in terms of fertilizing ability within males (Seidel, 1999). Mendel's law of independent assortment, including a 50:50 sex ratio requires phenotypic identity of genetically different sperm, which makes it very difficult to distinguish X-sperm from Y-sperm. As indicated earlier, bovine X-sperm have about $4 \%$ more total DNA than $\mathrm{Y}$-sperm. The X-sperm clearly are very slightly heavier on average than the Y-sperm, but distributions overlap, much like trying to sex humans by their height or weight. There is one convincing study using centrifugation to separate the smaller human Y-sperm from X-sperm (Koundouros and Verma, 2012), but their procedure is completely impractical plus the procedure almost certainly enriches for monosomies. It is the equivalent of saying humans more than 2 meters tall likely are men, but this excludes being able to assign sex to $>99 \%$ of men; this population also would have an excess of XYY men. There is one study using an immunological procedure for sexing bovine sperm that provides some evidence of efficacy (Sang et al., 2011). However, it also is impractical for a number of reasons and possibly only works for the $\mathrm{dead} /$ dying sperm in the ejaculate.

One other approach is to use transgenic technology to create sperm that are sexable. Hermann et al. (1999) have produced a strain of mice that naturally has a 2:1 male to female sex ratio in progeny without sorting sperm. Currently, however, there is a reluctance to invest in transgenic food-producing animals, and making such transgenic cattle would be very expensive.

Although currently available, commercial sexed semen is not yet an ideal product, but improvements will continue to be made. While I have emphasized how sexed semen is produced and used in the United States, the situation is very similar in Canada and several other countries.

\section{References}

Herrmann BG, Koschorz B, Wertz K, McLaughlin KJ and Kispert A 1999. Nature 402, 141-146.

Koundouros S and Verma P 2012. International Journal of Andrology 35, 880-886.

Sang L, Yang WC, Han L, Liang AX, Hua GH, Xiong JJ, Huo LJ and Yang LG 2011. Journal of Dairy Science 94, 20602070 .

Rath D, Barcikowski S, de Graaf S, Garrels W, Grossfeld R et al. 2013. Reproduction 145, R15-R30.

Seidel GE Jr 2012. Journal of Reproduction and Development 58, 505-509. 


\title{
Applications and cost benefits of sexed semen in pasture-based dairy production systems
}

\author{
S T Butler ${ }^{1}$ and I A. Hutchinson* \\ Teagasc, Animal and Grassland Research and Innovation Centre, Moorepark, Fermoy, Co. Cork, Ireland \\ ${ }^{*}$ Current address Nutribio, Tivoli Industrial Estate, Cork, Ireland
}

Email: stephen.butler@teagasc.ie

Implications When used under optimum conditions, sexed semen has the potential to add significant value in seasonal, pasture-based dairy production systems, both at farm and wider industry levels. It is likely that sexed semen will be used more widely as its fertility performance improves and the efficiency of the sorting procedure increases.

\begin{abstract}
Sexed semen technology is now commercially available in many countries around the world, and is primarily used in dairy cattle breeding. Sperm are sorted by flow cytometry on the basis of a $4 \%$ difference in DNA content between sperm containing X and Y chromosomes. Despite reliably producing a $90 \%$ gender bias, the fertility of the sexed semen product is compromised compared to conventional semen. The negative implications of the reduced fertility of sexed semen are amplified in seasonal systems of dairy production, as the importance of fertility is greater in these systems compared with year-round calving systems. A review of the literature indicates that conception rates to first service with frozen-thawed sexed semen are approximately $75 \%$ to $80 \%$ of those achieved with conventional frozen-thawed semen. Preliminary results from a large-scale field trial carried out in Ireland in 2013 suggest that significant improvements in the performance of sexed semen have been made, with conception rates of $87 \%$ of those achieved with conventional semen. The improved fertility of a sexed semen product that delivers a $90 \%$ gender bias has considerable implications for the future of breeding management in pasture-based dairy production systems. Sexed semen may facilitate faster, more profitable dairy herd expansion by increasing the number of dairy heifer replacements born. Biosecurity can be improved by maintaining a closed herd during the period of herd expansion. In a non-expansion scenario, sexed semen may be used to increase the value of beef output from the dairy herd. The replacement heifer requirements for a herd could be met by using sexed semen in the first 3-wk of the breeding season, with the remaining animals bred to beef sires, increasing the sale value over that of a dairy bull calf. Alternatively, very short gestation sires could be used to shorten the calving interval. Market prices have a considerable effect on the economics of sexed semen use, and widespread use of sexed semen should be restricted to well managed herds that already achieve acceptable herd fertility performance.
\end{abstract}

Conclusions and future outlook Sexed semen is now routinely used on heifers in many countries practising year-round calving. Preliminary results of a large scale field trial conducted in Ireland in 2013 indicate that the fertility of sexed semen, traditionally the barrier to extensive uptake of the technology in seasonal dairy systems, has improved significantly from previous reports in the literature. A sexed semen product that reliably produces $90 \%$ heifer calves with minimal reductions in fertility has the potential to transform breeding management in seasonal, pasture-based systems of milk production. Economic modelling has been utilised in a number of studies to evaluate the economic benefits of using sexed semen. Individual simulations examine specific scenarios that are often specific to an industry or production system. These simulations, however, provide important information on the factors that will have greatest influence over the financial success of sexed semen use. The fertility of the sexed semen product will be the critical issue determining the uptake of sexed semen in seasonal, pasture based systems. Further gains in the technical efficiency of the sorting process will continue to improve fertility, with subsequent effects on the uptake of the technology. Though dependant to some extent on market conditions, sexed semen has the potential to add significant value to seasonal, pasture-based dairy production systems, at both farm and industry level. 


\section{Improving bovine semen diluents: insights from the male and female reproductive tracts, and the potential relevance of cervical mucins}

J A McGetrick, C J Reid and S D Carrington

Veterinary Sciences Centre, University College Dublin, Belfield, Dublin 4, Ireland

Email: stephen.carrington@ucd.ie

Implications Artificial insemination is used for almost $20 \%$ of breeding cows worldwide. There is a need for semen extenders that enhance and prolong semen fertility beyond that which is possible using existing commercial formulations for cryopreservation or liquid semen storage. This could allow the use of reduced numbers of sperm per insemination, enhance the use of bulls of high genetic merit, and facilitate the greater application of fresh and sex-sorted semen. Here we review current information on the microenvironments of sperm as they mature and migrate. We also discuss how this knowledge can be used in the design of next-generation semen diluents.

Abstract The commercial applicability of bovine artificial insemination (AI) depends on the effectiveness of diluents for maintaining sperm fertility. Challenges faced by the AI industry due to recent advances in assisted reproduction, and the limitations inherent in using fresh and frozen-thawed sperm for AI, could be overcome with the development of better semen diluents. Research into the different microenvironments of bovine sperm as they progress towards maturity, capacitation, and fertilisation is revealing various mechanisms that could be exploited to improve the formulation of semen diluents. These are reviewed here. A rationale for a more detailed investigation of bovine cervical mucus for factors that may allow further progress towards this goal are also discussed.

Conclusion Many factors have been identified that contribute to the maintenance of sperm viability and enhancement of sperm fertility in the bovine male and female reproductive tract. These have the potential to be exploited in bovine semen extenders to overcome current issues in bovine AI relating to sperm quality and lifespan. Increased understanding of functionally relevant changes that sperm undergo at different developmental and migratory stages has arisen from research into the interaction between sperm and their different microenvironments. This knowledge has great potential to permit the design of extenders that prevent the premature capacitation of sperm and enhance the effectiveness of AI. Research into the interaction of bovine sperm with cervical mucus could lead to innovative diluents through insights gained into the regulation of sperm transit and sperm physiology. The environments experienced by sperm throughout their journey all influence the likelihood of successful fertilisation and greater understanding of the interactions between sperm and these microenvironments is helping to create a bright picture for the future of bovine semen diluents. 


\title{
Herd monitoring to optimise fertility in the dairy cow - making the most of herd records, metabolic profiling and ultrasonography (research into practice)
}

\author{
R F Smith ${ }^{1}$, J Oultram ${ }^{1}$, H Dobson ${ }^{1}$ \\ ${ }^{1}$ School of Veterinary Science, University of Liverpool, Leahurst Campus, Neston, Cheshire, CH64 7TE, UK \\ Email:robsmith@liv.ac.uk
}

Implications This review describes how dairy herd records, measurement of metabolic compounds and detection of the physiological state of a cows reproductive tract by ultrasonography can be used to monitor reproductive and nutritional management of a herd. It guides the selection of timely key performance indicators (KPIs) that monitor specific events in the period after calving and during the service period. It suggests the development of new KPIs to exploit research findings to improve on-farm performance. By deploying these findings farmers could improve cattle fertility and herd profitability.

\begin{abstract}
Fertility performance is intrinsically linked to the quality of the animal environment, overall management and nutrition. This review describes the use of dairy herd records, metabolic profiles and ultrasonographic findings at veterinary fertility examinations to monitor and manage dairy herd fertility. After calving, a cow has to overcome a series of physiological hurdles prior to establishing a pregnancy. The selection of timely key performance indicators (KPIs) that monitor specific events in the postpartum and service periods is vital to correctly identify problems and their potential causes that hopefully can be rectified. Cumulative sum ( $\mathrm{Cu}$-sum) charts are the timeliest monitors of efficiency of detection of oestrus, insemination outcome and relationship between postpartum events and fertility, with the point of inflection indicating when a change took place. Other KPIs use data from specific cohorts, adding an inherent delay to when change is indicated. Metabolic profiles and milk constituent data allow monitoring of nutritional adequacy and developments to offer new possibilities of on-farm systems for regular measurements of milk constituents (including progesterone) and energy status. Examination of the reproductive tract can be used to indicate individual and herd fertility status but the currently available detail is under used. Recent advances in ultrasonography can improve the diagnosis of reproductive tract pathophysiology still further but the clinical use of these methods in veterinary practice needs further evaluation. Development of new KPIs to exploit research findings are needed to ensure this knowledge is used to improve on-farm performance.

Conclusion Fertility is intrinsically linked with milk production and profitability. Fertility performance is a bellwether for quality of the animal environment, overall management and nutrition. To have a positive influence on fertility the farm management team of herd manager, agricultural advisor, nutritionist and veterinary surgeon need to actively monitor up-todate information. Selecting the correct KPIs for the farming system employed and the data available are keys to this task. As our understanding of postpartum (patho-)physiology develops new KPIs need to be developed to allow farmers and veterinary surgeons to monitor animals during this key period.
\end{abstract}


Use of herd management programmes to improve reproductive performance of dairy cattle $\mathrm{S} \mathrm{McDougall}^{1}$, C Heuer ${ }^{2}, \mathrm{~J} \mathrm{Morton}^{3}$, T Brownlie ${ }^{1, \mathrm{a}}$

${ }^{1}$ Cognosco, Anexa Animal Health, PO Box 21 Morrinsville, 3300, New Zealand

${ }^{2}$ Epicentre, IVABS, Massey University, Palmerston North, 4474, New Zealand

${ }^{3}$ Jemora Pty Ltd, PO Box 2277, Geelong, 3220, Victoria, Australia

${ }^{a}$ Present address: LIC, Private Bag 3016, Hamilton, 3240, New Zealand_Email: smcdougall@anexa.co.nz

Implications A planned animal health approach to dairy herd fertility was demonstrated, via a controlled randomised study, to improve fertility at herd level. The programme tested (InCalf) used a management cycle approach, i.e. assessment of current performance against goals and objectives, identification of areas for improvement, development and implementation of a plan and review. Such an approach allowed identification of the economically important areas for improvement. Success of this approach provides industry leaders and service providers (e.g. veterinarians and other rural professionals) with confidence that if appropriately implemented, such planned animal health services do have positive effects on herd performance.

Abstract There has been a long history of herd health and production management programmes in many dairy industries around the world, but evidence for the efficacy of such programmes is limited. In response to a perceived decline in fertility of dairy cows, a herd reproductive management programme (InCalf) was introduced to New Zealand in 2007. This programme uses a management cycle approach that includes an assessment of the current herd status, identification of areas for improvement, development of a plan, implementation of this plan and finally a review process. The programme uses facilitators who work with farmers either in a one-to-one manner or in a formalised group setting that involves a series of meetings over a 12 month period (the farmer action group). The hypothesis that involvement in a reproductive management programme would improve herd reproductive performance was tested using a herd-level controlled randomised study (the National Herd Fertility Study) involving herds in four geographic regions of New Zealand over 2 years. Within each region herds were ranked on the 6-week in-calf rate (i.e. the proportion of the herd pregnant in the first 6 weeks of the seasonal breeding programme) in the year preceding commencement of the study, then randomly assigned to be involved in a farmer action group or left as untreated controls. The key outcome variable of the study was the 6-week in calf rate. Pregnancy diagnosis was undertaken at 12 weeks after the start of seasonal breeding programme which allowed determination of conception dates and hence calculation of the 6-week in-calf rate. Additionally measurements including heifer live weight, and body condition score (pre-calving and pre-mating) were undertaken to test whether treatment resulted in measurable changes in some of the key determinants of herd reproductive performance. Involvement in the farmer action group of InCalf resulted in a 2 percentage point increase in 6 -week in-calf rate $(P=0.05)$. Additionally in herds involved in the farmer action group, heifers had live weight closer to target, body condition score of cows pre-mating was higher, and oestrous detection rates were higher. It was concluded that involvement in this herd reproductive management programme improved reproductive outcomes in this New Zealand study. However to achieve substantial improvements in herd reproductive performance at regional or national level a greater response to the programme and a high uptake of such programmes is required as well as use of other industry level tools such as genetic management programmes.

Conclusions Planned animal health and production management programmes have a long history. They have evolved from simple monthly visits to farms examining a selected category of cows through to management cycle based approaches taking a holistic view of reproduction within the farm system. The introduction of the InCalf programme to New Zealand in 2007 provided an opportunity to test whether such programmes do actually improve reproductive outcomes in commercial farms, and to examine the mechanism by which the programme worked. Based on a large, herd-level randomised controlled study, we concluded that participation in an InCalf farmer action group had a small positive effect on herd reproductive performance. However, for the New Zealand dairy industry to achieve its stated goal of having half the herds nationally achieving 6-week in-calf rates of $>78 \%$ by 2020 , not only would the majority of herds nationally need to be enrolled in InCalf, but those herds would need to achieve a substantially greater increase in 6-week in-calf rate than 2 percentage points per annum.

Clearly improvements in the uptake and effectiveness of herd reproductive performance programmes are required, including improvements in the technical approach, substantial marketing efforts, and improved training and implementation of whole farm assessment and facilitation skills by rural professionals. Incorporation of the new insights into on-farm change provided by social science models need to be more generally understood by those providing services on farm and to be incorporated in the design of such programmes. Many of the same barriers to implementation of on-farm change also apply to the veterinary and extension businesses providing such services. Veterinarians are recognised as key providers of technical advice to farmers around fertility and as such are the logical providers of programmes designed to improve reproductive performance. However due to the competing opportunities within the businesses of rural professionals, provision of herd health programmes must be as profitable, if not more so, than other activities. 


\title{
Economics of fertility in high-yielding dairy cows on confined TMR systems
}

\section{E Cabrera}

Department of Dairy Science, University of Wisconsin-Madison, 1675 Observatory Dr., Madison, WI 53706, USA. Email:vcabrera@wisc.edu

Implications High-yielding dairy cows on confined TMR systems improve their profitability as they improve reproductive performance, which determines higher milk production, more calves, lower replacement costs, and lower relative reproduction costs. Overall reproductive performance can be measured by average pregnancy rate in 21-day intervals, which ranges between 10 and 40\%. Most farms rely on a combination of oestrous detection and synchronisation ovulation control programme, which seems to be economically effective. Use of alternative pregnancy diagnosis such as earlier blood chemical tests could be economically feasible if accurate. Opportunities for cow-level reproductive management exist within herd defined reproductive programmes.

\begin{abstract}
The objective of this review paper was to summarise the latest findings in dairy cattle reproductive economics with an emphasis on high-yielding, confined TMR systems. The economic gain increases as the reproductive efficiency improves. These increments follow the law of diminishing returns, but are still positive even at high reproductive performance. Reproductive improvement results in higher milk productivity and, therefore, higher milk income over feed cost, more calf sales and lower culling and breeding expenses. Most high-yielding herds in the USA use a combination of timed artificial insemination (TAI) and oestrous detection (OD) reproductive programme. The ratio of achievable pregnancies between OD and TAI determines the economic value difference between both and their combinations. Nonetheless, complex interactions between reproductive programme, herd relative milk yield, and type of reproductive programme are reported. For example, higher herd relative milk yield would favour programme relying more on TAI. In addition, improved reproductive efficiency produces extra replacements. The availability of additional replacements could allow more aggressive culling policies (e.g., less services for non-pregnant cows) to balance on-farm supply and demand of replacements. Balancing heifer replacement availability in an efficient reproductive programme brings additional economic benefits. New technologies such as the use of earlier chemical tests for pregnancy diagnosis could be economically effective depending on the goals and characteristics of the farm. Opportunities for individual cow reproductive management within defined reproductive programme exist. These decisions would be based on economic metrics derived from the value of a cow such as the value of a new pregnancy, the cost of a pregnancy loss, or the cost of an extra day open.
\end{abstract}

Conclusions Improved reproductive performance increases overall herd economic net returns. Economic factors that contribute to it are higher milk sales, calf sales, lower replacement costs, and lower relative reproductive costs. Most highyielding TMR dairy farms use a combined approach that includes synchronisation protocols and oestrous detection interventions in their reproductive programmes. The reproductive performance on these farms can be measured and compared using the standard 21-day PR, which captures the interacting reproductive performance of these combined programmes. With an observed 21-day PR of between 10 and 40\%, the economic net gain increases as the pregnancy rate increases. Within those reproductive programmes, the use of blood chemical pregnancy diagnoses one-week earlier that a conventional pregnancy diagnosis, can be economically effective as long the test is more than $94 \%$ accurate and the herd records indicate that the early pregnancy losses do not exceed $10 \%$. Using the economic value of a cow or its equivalent RPO to perform individual cow fine-tuning of reproductive decisions within defined herd reproductive programmes would improve reproductive performance and in turn net returns. Decision support tools to evaluate the economic outcomes of reproductive programmes and cow-level reproductive decisions in high-yielding dairy farms are available at the University of Florida (http://dairy.ifas.ufl.edu/tools/) and at the University of Wisconsin (http://dairymgt.uwex.edu/tools.php). 


\title{
Effect of fertility on the economics of pasture-based dairy systems
}

\author{
L Shalloo $^{1}$, A. Cromie $^{2}$ and $\mathrm{N} \mathrm{McHugh}^{1}$ \\ ${ }^{1}$ Teagasc, Animal \& Grassland Research and Innovation Centre, Moorepark, Fermoy, Co. Cork, Ireland \\ ${ }^{2}$ Irish Cattle Breeding Federation, Bandon, Co. Cork, Ireland \\ Email: Laurence.Shalloo@Teagasc.ie
}

Implications The overall costs associated with reproductive inefficiency are larger in pasture-based systems driven by the potential reduction in the synchrony between feed supply and feed demand at farm level due to potentially increased calving intervals. Key traits affecting the economics of overall fertility are calving interval, survivability, level of AI use and intervention and overall labour efficiency. Identifying and communicating key simple economic messages (six week calving rate) around key performance indicators facilitate a targeted response to increasing fertility on the farm.

\begin{abstract}
There are significant costs associated with reproductive inefficiency in pasture-based dairy herds. This study has quantified the economic effect of a number of key variables associated with reproductive inefficiency in a dairy herd and related them to six week calving rate for both cows and heifers. These variables include: increased culling costs, the effects of sub optimum calving dates, increased labour costs and increased AI and intervention costs. The Moorepark Dairy Systems Model (MDSM) which is a stochastic budgetary simulation model was used to simulate the overall economic effect at farm level. The effect of change in each of the components was simulated in the model and the costs associated with each component was quantified. An analysis of national data across a four year period using the Irish Cattle Breeding Federation (ICBF) database was used to quantify the relationship between the six week calving rate of a herd with survivability (\%), calving interval (days) and the level of AI usage. The costs associated with increased culling (\%), calving date slippage (day), increased AI and intervention costs ( 0.1 additional inseminations), as well as, increased labour costs $(10 \%)$ were quantified as $€ 13.68, € 3.86$, €4.56 and €29.6/cow/year. There was a statistically significant association between the six week calving rate and survivability, calving interval and AI usage at farm level. A $1 \%$ change in six week calving rate was associated with $€ 9.26$ /cow/annum for cows and $€ 3.51 /$ heifer/annum for heifers. This study does not include the indirect costs such as reduced potential for expansion, increased costs associated with failing to maintain a closed herd as well as the unrealised potential within the herd.
\end{abstract}

Conclusion There are significant costs associated with reproductive inefficiency in the dairy herd. This study has quantified the costs associated with increased culling, calving date slippage, increased AI and intervention costs as well as increased labour costs. This study does not include the indirect costs such as reduced potential for expansion, increased costs associated with not having a closed herd as well as the unrealised potential within the herd. Analysis of the Irish national database has shown that there is a statistically significant association between the six week calving rate and survivability, calving interval and AI usage at farm level. Each 1\% change in six week calving rate was associated with $€ 8.22$ /cow/annum for a herd average cow in the dairy herd. 